\title{
VLBI Measurements for Frequency Transfer
}

\author{
Hiroshi Takiguchi, Yasuhiro Koyama, Ryuichi Ichikawa, \\ Tadahiro Gotoh, Atsutoshi Ishii, Thomas Hobiger, \\ and Mizuhiko Hosokawa \\ National Institute of Information and Communications Technlogy, Japan \\ email: htaki@nict.go.jp
}

\begin{abstract}
We carried out the intercomparison experiments between VLBI, GPS and DMTD to show the VLBI can measure the right time difference. We produced the artificial change using by line stretcher. At the artificial change part, VLBI and DMTD show good agreement, less than 10ps. The quantity and sense of VLBI results match well with DMTD results. Consequently, the geodetic VLBI technique can measure the right time difference.
\end{abstract}

Keywords. time, VLBI, time and frequency transfer.

As one of the new frequency transfer technique to compare the next high stable frequency standards, we proposed the geodetic VLBI technique. Previously, we evaluated the ability of VLBI frequency transfer by comparison with GPS carrier phase frequency transfer using data from IVS and IGS. The results of the VLBI showed that the stability follows a $1 / \tau$ law closely and it surpassed the stability of atomic fountain at about $10^{3}$ seconds. And that showed the stability has reached about $2 \times 10^{-11}$ at 1 sec. These results showed that geodetic VLBI technique has the potential for precise frequency transfer.

Furthermore, to show the capability of VLBI, we carried out the intercomparison experiments between VLBI, GPS and DMTD at Kashima34m-Kashima11m baseline. We inserted two line stretchers at the route of the reference signal from Hydrogen maser to Kashima11m antenna to change time delay artificially. Usually, geodetic VLBI observe the multiple sources that covered uniformly the sky alternately. And we usually estimate clock, atmosphere and station coordinates at the time of analysis. However in this experiment, we observed only one source, and we estimated only clock parameters.We employed CALC/SOLVE and NRC's PPP to analyze VLBI and GPS respectively.

The result of intercomparison between GPS and DMTD shows that there are the parts of good agreement. But at the artificial change parts, GPS show the opposite sense and large difference from DMTD. The result of intercomparison between VLBI and DMTD shows that, at the artificial change parts, VLBI and DMTD show good agreement. The quantity and sense of VLBI results match well with DMTD. For example, at one of the artificial change part, the difference between GPS and DMTD is 26ps. On the other hand, the difference between VLBI and DMTD is only 7ps. Almost all the differences between VLBI and DMTD at the artificial change parts are less than about 10ps. At other parts, VLBI shows good agreement less than 50ps for short time range, but larger difference for longer time range due to the effect of atmospheric variation. The result of our experiment clearly shows that the geodetic VLBI technique can measure the right time difference.

\section{Acknowledgements}

We would like to acknowledge IVS and IGS for the products. We are grateful that GSFC and NRC provided the analysis software. 